
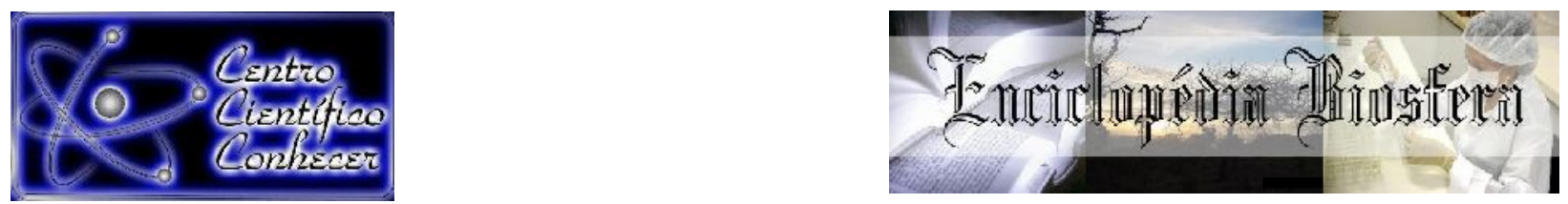

\title{
CARCINOMA PAPILÍFERO DE GLÂNDULA CERUMINOSA EM UM FELINO DOMÉSTICO: RELATO DE CASO
}

Sheila Santana de Mello Luis Filipe Pateis Ferreira1; Lorena Poliana Rodrigues Gonçalves; Breno Almeida Wanderley3; Thaisa Reis dos Santos ${ }^{4}$; Guilherme Nascimento Cunha ${ }^{5}$

${ }^{1}$ Graduandos em Medicina veterinária pelo Centro Universitário de Patos de Minas- UNIPAM, Patos de Minas-MG.

Especialista em Clínica e Cirurgia de Pequenos Animais, Dermatologia e Clínica médica de Felinos pela faculdade Qualittas.

${ }^{3}$ Mestre em Sanidade e produção animal nos trópicos pela Universidade de Uberaba, docente no Centro Unniversitário de Patos de Minas - UNIPAM.

${ }^{4}$ Doutora em Ciências Veterinárias na área de Saúde Animal pela Universidade

Federal de Uberlândia, docente na Universidade federal de Uberlândia.

${ }^{5}$ Doutor em Cirurgia Veterinária pela Universidade Estadual Paulista Júlio de Mesquita Filho, docente no Centro Universitário de Patos de Minas - UNIPAM. E-mail: sheilamellovet@gmail.com

Recebido em: 15/11/2021 - Aprovado em: 15/12/2021 - Publicado em: 30/12/2021 DOI: 10.18677/EnciBio_2021D13

\section{RESUMO}

Afecções nas glândulas ceruminosas presentes no meato acústico externo são raras e o prognóstico pode variar de favorável a desfavorável. Dentre estas, têm-se cistos de glândulas ceruminosas, hiperplasia glandular, adenoma e adenocarcinoma em gatos. O presente trabalho teve como objetivo relatar o caso de um felino doméstico diagnosticado com carcinoma papilífero de glândula ceruminosa. Foi atendida uma gata, sem raça definida, 11 anos com histórico de otite recorrente, diagnosticada com Carcinoma Papilífero de Glândula Ceruminosa através do exame histopatológico. O animal foi submetido a um procedimento cirúrgico de ablação total do conduto auditivo e a neoplasia foi removida em sua totalidade. No pós-operatório imediato o animal apresentou protrusão de terceira pálpebra e paralisia palpebral, ambas do lado ipsilateral ao conduto auditivo excisado, além de ataxia, devido possível lesão do nervo facial. Estas alterações foram temporárias, entretanto, após dois meses do procedimento cirúrgico o felino teve como sequela o aparecimento intermitente de "Head tilt" e queda da pina permanente. Após 60 dias do procedimento cirúrgico o paciente foi reavaliado e permanecia sem sinal de recidiva da neoplasia. Conclui-se que o carcinoma papilífero de glândulas ceruminosas deve estar entre os diagnósticos diferenciais de pacientes felinos com histórico de otite crônica e reforça a importância da realização da otoscopia para análise do conduto auditivo de pacientes com otite crônica. A ablação total de conduto auditivo se mostra eficaz para o tratamento do carcinoma papilífero de glândulas ceruminosas.

PALAVRAS-CHAVE: Cerúmen. Gato. Neoplasia. 


\title{
PAPILLARY CARCINOMA OF CERUMINOUS GLANDS IN A DOMESTIC FELINE: A CASE REPORT
}

\begin{abstract}
Affections in the ceruminous glands present in the external acoustic meatus are rare and the prognosis can vary from favorable to unfavorable. Among these, there are ceruminous gland cysts, glandular hyperplasia, adenoma and adenocarcinoma in cats. The present work aimed to report the case of a domestic feline diagnosed with papillary carcinoma of the ceruminous gland. An 11-year-old, mixed-breed cat with a history of recurrent otitis, diagnosed with Papillary Carcinoma of the Ceruminous Gland was seen through histopathological examination. The animal underwent a surgical procedure for total ablation of the auditory meatus and the neoplasm was removed in its entirety. In the immediate postoperative period, the animal presented third eyelid protrusion and palpebral palsy, both on the side ipsilateral to the excised auditory meatus, in addition to ataxia, due to possible injury to the facial nerve. These alterations were temporary, however, after two months of the surgical procedure, the feline had the intermittent appearance of "Head tilt" and permanent pinna fall as a sequel. After 60 days of the surgical procedure, the patient was re-evaluated and there was no sign of recurrence of the neoplasm. It is concluded that papillary carcinoma of the ceruminous glands should be among the differential diagnoses of feline patients with a history of chronic otitis and reinforces the importance of performing otoscopy to analyze the auditory meatus of patients with chronic otitis. Total ablation of the auditory meatus is effective for the treatment of papillary carcinoma of the ceruminous glands.
\end{abstract}

KEYWORDS: Cerumen. Cat. Neoplasm

\section{INTRODUÇÃO}

Afecções nas glândulas ceruminosas são raras, e o prognóstico pode variar de favorável a desfavorável. Algumas anormalidades já foram relatadas, dentre elas cistos de glândulas ceruminosas em gatos, hiperplasia glandular, adenoma e adenocarcinoma, sendo que os dois últimos ocasionalmente são relatados em cães e gatos. A incidência desses tumores não é conhecida, no entanto, com base em levantamentos de envios aos laboratórios de patologia animal, de todos os tumores que afetam felinos a porcentagem relativa àqueles do canal auditivo é inferior a $2 \%$ (KUBBA et al., 2018).

As causas que desencadeiam esses tumores ainda não foram totalmente elucidadas. Devido aos relatos de ocorrência dos mesmos após episódios de otite externa, acredita-se que existe relação entre processos inflamatórios crônicos e o aparecimento de lesões no conduto auditivo de felinos. A agressão causada pelo animal frente ao prurido, a produção e consequente retenção de cera pelas glândulas ceruminosas da orelha são alguns fatores que influenciam a carcinogênese. Também já foram associadas à própria anatomia da orelha externa, bem como à conduta terapêutica incorreta que pode levar ao agravamento do quadro, a perda auditiva e até mesmo auxiliar no desenvolvimento de neoplasias (COSTA et al., 2018).

Adenocarcinomas em conduto auditivo de felinos acometem com mais frequência animais geriátricos de 9 a 10 anos de idade e não possuem predileção por sexo. A sintomatologia clínica geralmente se assemelha aos sintomas clássicos de otite crônica. Na maioria das vezes somente uma orelha é afetada (DIAZ, 2018).

Suspeita-se da ocorrência de neoplasia a partir da otoscopia, exame que 
permite a visualização de massas no conduto auditivo externo, associado à sintomatologia apresentada pelo animal (sacudir a cabeça, coçar as orelhas, atorreia, odor fétido, geralmente otite bacteriana externa secundária e, por vezes, sangramento do ouvido afetado). O diagnóstico definitivo ocorre por meio da citologia ou histopatológico da lesão, sendo este último o exame que determina o diagnóstico definitivo da neoplasia. Ressalta-se que, diante da suspeita de lesões neoplásicas, a realização de exames de imagens é importante para o estadiamento clínico do paciente, pesquisa de metástases em órgãos distantes, bem como determinar o prognóstico do animal (MEDEIROS et al., 2014).

O tratamento de eleição é o cirúrgico, sendo que existem duas técnicas disponíveis, a ressecção lateral do canal auditivo ou a ablação total do conduto auditivo. A segunda técnica possui melhores taxas de sucesso, tendo em vista que a ressecção lateral do canal auditivo possui até $70 \%$ de chances de recidivas (GRANDII; RONDELLI, 2017). O presente estudo teve como objetivo relatar o caso de um felino doméstico diagnosticado com carcinoma papilífero de glândula ceruminosa após sucessivos episódios de otite externa.

\section{RELATO DE CASO}

Foi atendido em uma clínica veterinária, um felino, fêmea, sem raça definida, tricolor, 11 anos, pesando $3,7 \mathrm{Kg}$ com histórico de otite recorrente. A tutora relatou que estava tratando há dois anos, sendo feito o revezamento dos medicamentos comerciais Oto sana (gentamicina, cetoconazol e valerato de betametasona) (Mundo animal, Brasil, Pindamonhangaba- SP) e Natalene (diazinon, pimaricina, neomicina e acetato de dexametasona) (Virbac, Brasil, Sorocaba-SP). No entanto, nenhum dos fármacos estava promovendo evolução positiva da afecção.

No exame físico notou-se áreas de alopecia na região cervical e na altura da escápula, resultante do ato de se coçar frequente. Por meio da otoscopia foi possível observar grande quantidade de secreção mucopurulenta no conduto auditivo direito e pela inspeção notou-se odor fétido. Prescreveu-se um veículo otológico manipulado a base de dexametasona a $1 \%$ e ciprofloxacina a $0,35 \%$ que resultou em um alívio considerável e temporário dos sintomas. Neste período foi feito acompanhamento clínico do animal utilizando esse mesmo tratamento.

Após 17 meses a paciente apresentou-se para consulta novamente com o quadro clínico de otite. Durante exame físico especifico, ao realizar a otoscopia observou-se a presença de várias massas menores que um $\mathrm{cm}$ de diâmetro, de coloração brancacenta no interior do conduto auditivo direito. A paciente foi encaminhada para a realização de procedimento de biopsia incisional, após realização de exames laboratoriais prévios e anestesia geral de acordo com procedimentos determinados pela clínica veterinária.

Antes da coleta das amostras, realizou-se swab otológico com cotonete estéril para a realização de exame de cultura e antibiograma. Após isto, coletados três fragmentos de $0,3 \mathrm{~cm}$ cada, acondicionados em frascos contendo formol a $10 \%$ e as amostras foram encaminhadas a um laboratório de patologia animal.

O exame histopatológico revelou neoplasia maligna caracterizada por proliferação papilífera de células com moderado pleomorfismo nuclear, núcleos ovalados, nucléolo central exuberante e citoplasma eosinofílico, presença de numerosas mitoses e estroma fibroso (Figura 01). Obteve-se como resultado diagnóstico "carcinoma papilífero de glândula ceruminosa" com características de malignidade, seguido de uma observação do laboratório informando que essa 
afecção era de ocorrência rara.

Quanto à cultura e antibiograma o resultado foi 0 isolamento de Staphylococcus $s p$, resistente a 14 antibióticos e sensível a nove. Baseando no resultado obtido prescreveu-se um veículo otológico a base de amicacina a $2 \%$ utilizando 5 gotas a cada 12 horas por sete dias, que auxiliou no controle da infecção.

Devido ao diagnóstico de carcinoma, a paciente foi encaminhada para a realização de exérese da lesão, mas o procedimento não foi realizado pelo tutor. Meses depois a paciente retornou apresentando progressão da lesão e aumento da massa e foi novamente encaminhada para realização de cirurgia. Devido à elevada tumefação foram administrados piroxicam $0,3 \mathrm{mg} / \mathrm{kg}$, via oral, a cada 48 horas por cinco dias e cefovecina sódica $8 \mathrm{mg} / \mathrm{kg}$, via subcutânea, dose única. Porém não foi observada remissão da massa e a paciente foi submetida a técnica de ablação total de conduto auditivo. Antes do procedimento cirúrgico foi realizada radiografia torácica nas posições latero-lateral direita, esquerda e ventro-dorsal e não foi evidenciada metástase pulmonar (Figura 02).

FIGURA 1- Microfotografia do epitélio do meato acústico da orelha externa direita. Setas mostram células com moderado pleomorfismo nuclear, núcleos ovalados, nucléolo central exuberante e citoplasma eosinofílico "carcinoma papilífero de glândulas ceruminosas". Aumento 40x

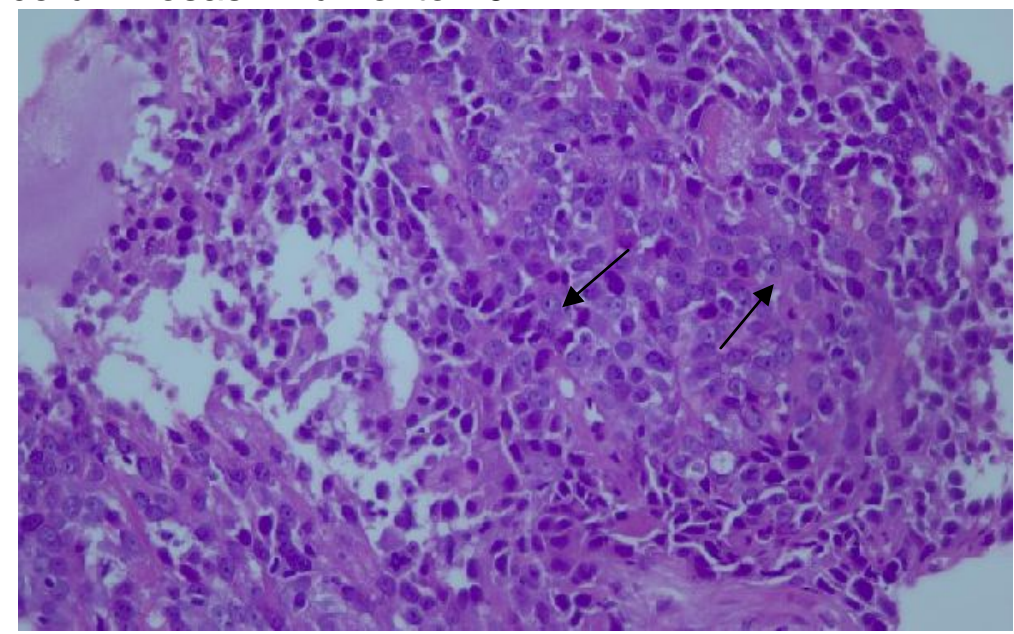

Fonte: Autores, (2019).

Foi realizada a ablação total do conduto auditivo. No pós-operatório imediato o animal apresentou temporariamente protrusão de terceira pálpebra e paralisia palpebral, ambas do lado ipsilateral ao conduto auditivo excisado, além de perda de equilíbrio. Após o procedimento cirúrgico o animal permaneceu em internação, foram administrados ciproeptadina $(0,5 \mathrm{mg} / \mathrm{kg}$, VO, duas vezes ao dia, por três dias), prednisolona $(0,5 \mathrm{mg} / \mathrm{kg}$, VO, uma vez ao dia, por 5 dias), dipirona $(25 \mathrm{mg} / \mathrm{kg}, \mathrm{VO}$, três vezes ao dia, por três dias), cloridrato de tramadol $(2 \mathrm{mg} / \mathrm{kg}, \mathrm{VO}$, três vezes ao dia, por cinco dias), cefovecina sódica ( $8 \mathrm{mg} / \mathrm{kg}$, SC, dose única) e mirtazapina (3,75 mg/animal, $\mathrm{VO}$, em dias alternados completando um total de cinco doses em 10 dias). 
FIGURA 2 - Radiografia torácica (Latero-lateral esquerda) sugerindo ausência de metástases

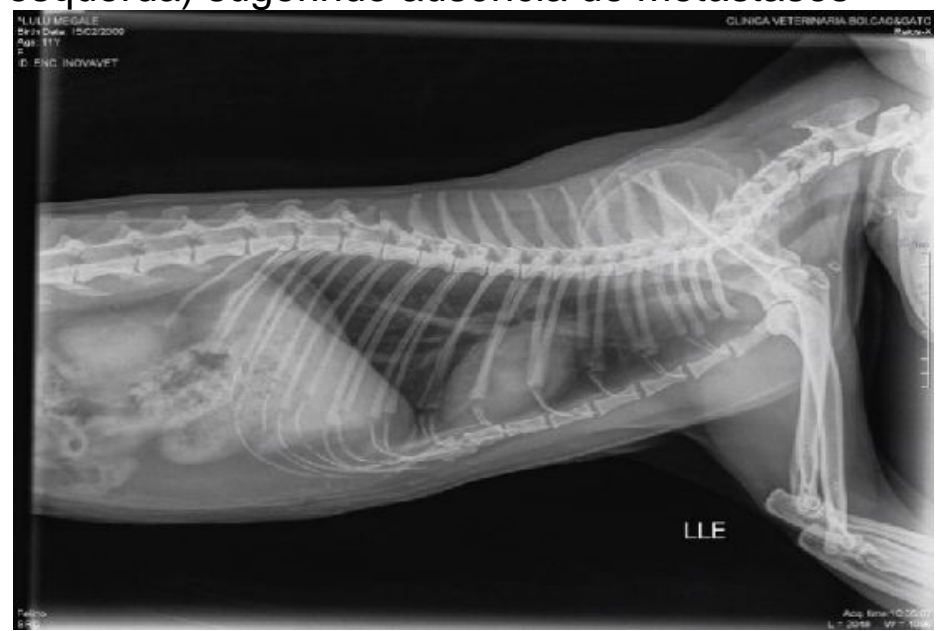

Fonte: Autores, (2020).

Dez dias após o procedimento cirúrgico ocorreu deiscência da sutura e foi realizado tratamento da ferida para cicatrização de segunda intenção. Para isso utilizou-se amoxilina + clavulanato de potássio $(12,5 \mathrm{mg} / \mathrm{kg}$, VO, duas vezes ao dia, por sete dias), cefovecina sódica (8mg/kg, SC, dose única) e também rifamicina tópica em spray (BID, por sete dias). A bioquímica sérica e hemograma não apresentaram alterações dignas de nota.

Após dois meses do procedimento cirúrgico o equilíbrio foi retomado, apesar disso, o animal teve como sequela o aparecimento intermitente de "Head till" ou inclinações da cabeça, e queda da pina permanente (Figura 3). Porém, sem nenhum sinal de recidiva da neoplasia.

FIGURA 3 - "Queda" permanente da pina, lado direito, após ablação total do meato auditivo.

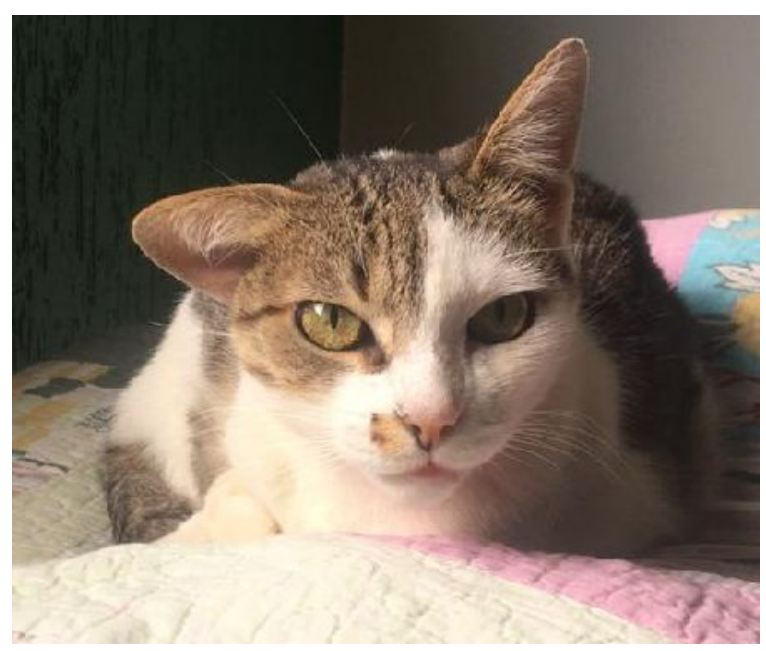

Fonte: Autores, (2020). 


\section{RESULTADOS E DISCUSSÃO}

O presente trabalho tratou-se de um relato de caso e foi aprovado pela Comissão de Ética no Uso de Animais (CEUA) do Centro Universitário de Patos de Minas - UNIPAM, sob protocolo 85/21. Este estudo visou relatar um caso raro em felinos. Poucos casos de carcinoma de glândula ceruminosa em humanos e animais domésticos têm sido descritos na literatura. Esse número é ainda menor quando relacionados a casos específicos e isolados em felinos domésticos. Importante salientar que de todos os relatos encontrados dessa neoplasia em gatos nenhum recebeu a denominação "papilífera" em sua descrição (BACON et al., 2003; MEDEIROS et al., 2014; COSTA et al., 2018; KUBBA et al., 2018),

Dentre estes relatos tem-se o de Bacon et al. (2003), no qual realizaram um estudo retrospectivo avaliando 44 procedimentos cirúrgicos de ablação total do canal auditivo em felinos. Destes, 37 possuíam neoplasias no conduto auditivo, sendo que 18 foram diagnosticados com adenocarcinoma de glândulas ceruminosas.

Neste relato, a paciente se tratava de uma fêmea felina diagnosticada com carcinoma de glândulas ceruminosas semelhante aos relatos de Medeiros et al. (2014) e Kubba et al. (2018), nos quais foram descritos, em cada trabalho, um caso clínico de uma gata com diagnóstico de carcinoma de glândula ceruminosa. Atualmente, o estudo mais recente foi o realizado por Pavletic (2019) que descreveu dois felinos machos avaliados com lesões cutâneas obstrutivas no conduto auditivo externo esquerdo. Um recebeu o diagnóstico de "cistomatose ceruminosa com colonização de superfície de levedura" compatível com Malassezia spp., e o outros "múltiplos adenomas ulcerados de glândula ceruminosa".

Ambos os estudos supracitados relataram otite externa secundária à obstrução do meato acústico e receberam como tratamento a ressecção parcial do terço superior do canal auditivo vertical afetado. Houve recidiva após um ano do procedimento cirúrgico no gato com adenoma da glândula ceruminosa. Fato este diferente da gata do atual estudo, a qual não evoluiu desfavoravelmente, com recidiva, até o momento. Provavelmente devido ao procedimento de ablação total do conduto auditivo afetado realizado no presente relato.

No presente estudo a gata acometida apresentava-se com 11 anos, sendo esta a mesma idade do caso de uma gata relatada por Medeiros et al. (2014). Kubba et al. (2018) descreveram a ocorrência desta neoplasia em uma gata com nove anos, idade inferior aos estudos supracitados. No entanto, apesar da pequena diferença entre a idade dos animais, é importante salientar que em todos os casos a neoplasia desenvolveu-se em animais idosos, acima de oito anos. Este achado é reforçado por Grandii e Rondelli (2017), os quais afirmam que os adenocarcinomas são mais comuns em felinos de sete a treze anos de idade.

Em relação ao sexo, apesar do presente relato descrever uma fêmea felina, Grandii e Rondelli (2017) afirmam a inexistência de predisposição sexual da doença. Corroborando este achado, o estudo realizado por Bacon et al. (2003), em que foram avaliados 28 animais do sexo masculino e 16 do sexo feminino, não foi constatado que o sexo interferiu na ocorrência da neoplasia.

Quanto ao aspecto macroscópico da neoplasia, este se apresentava como uma massa milimétrica, ulcerada, de consistência dura, que obstruiu parcialmente o conduto auditivo externo de ambos os lados. Medeiros et al. (2014), citam em seu estudo a presença de uma neoformação friável e que possuía consistência macia, coloração vermelha, irregular, com superfície ulcerada e aderência aos planos profundos. 
A paciente em questão teve histórico de otite crônica recorrente. Ao analisar a ocorrência de otite recorrente como causa primária e predisponente das neoplasias localizadas no conduto auditivo dos felinos, Grandii e Rondelli (2017) reforçam esta afirmação, uma vez que alterações genéticas podem ser advindas de traumas e processos inflamatórios reincidentes. Geralmente, alguns sinais clínicos que podem ser observados são odor fétido, prurido, inclinação da cabeça para o lado afetado, agitação da cabeça, e em casos mais avançados pode haver também a presença de exsudato, por vezes hemorrágico (DIAZ, 2018).

Este relato ressalta que o histórico de otite recorrente serve como alerta para a importância da realização da otoscopia, que deve ser realizada sempre que houver queixa de qualquer alteração em ouvidos. A ausência da realização deste exame, bem como, a presença de inflamação do conduto e secreção podem dificultar a visualização da massa e, consequentemente, retardar o diagnóstico.

Por fim, quanto à cirurgia como tratamento de escolha para a maioria dos tumores localizados em conduto auditivo, Grandii e Rondelli (2017) consideram a ablação total do conduto auditivo o procedimento cirúrgico padrão ouro, uma vez que a taxa de recidiva em gatos submetidos à ressecção lateral do conduto auditivo é de até $70 \%$. Estes argumentos são sustentados até então pela evolução do quadro da gata do atual relato, uma vez que a paciente se encontrava sem sinal de recidiva da lesão após dois meses do procedimento cirúrgico.

Diaz (2018) relata que o uso de quimioterapia sistêmica em pacientes com estado mais agressivo ou avançado de tumor de glândulas ceruminosas não é eficaz. Portanto, não foi realizada neste caso, e embora o diagnóstico tenha sido tardio a paciente apresentou benefício clínico apenas com o procedimento cirúrgico.

Ao avaliar o período pós-cirúrgico a gata apresentou incoordenação motora, protusão de terceira pálpebra e paralisia palpebral temporárias, queda da pina permanente (ambas do lado do conduto auditivo excisado), e o aparecimento esporádico de "Head tilt" ou "inclinações da cabeça", o que pode indicar lesões na inervação local durante a cirurgia. Provavelmente o nervo vestibulococlear sofreu injúria devido a presença de incoordenação e inclinações da cabeça (NELSON, COUTO; 2021). Além disso, ramos do nervo facial podem ter sido lesionados neste caso, pois Taylor (2006) afirma que a queda da pina é um dos sinais clínicos nestas situações como resultado da perda do tônus muscular do lado acometido.

Embora a paciente tenha apresentado sequela permanente com alteração de orelha supracitada, após dois meses do procedimento a paciente foi avaliada e encontrava-se em um bom estado geral, sem sinal de otite e sem evidência de recidiva da lesão, ressaltando a eficácia da ablação total de conduto auditivo como tratamento do carcinoma papilífero de glândula ceruminosa em ouvido.

\section{CONCLUSÃO}

Conclui-se que o carcinoma papilífero de glândulas ceruminosas deve estar entre os diagnósticos diferenciais de pacientes felinos com histórico de otite crônica e reforça-se a importância da realização da otoscopia para análise do conduto auditivo de pacientes com otite crônica. A ablação de conduto auditivo se mostra eficaz para o tratamento do carcinoma papilífero de glândulas ceruminosas.

\section{REFERÊNCIAS}

BACON, N. J.; GILBERT, R. L.; BOSTOCK, D. E.; WHITE, R. A. S. Total ear canal ablation in the cat: indications, morbidity and long-term survival. Journal of small animal practice, OCTOBER, v. 44, n.10, p. 430-434., 2003. Disponível em: < 
https://pubag.nal.usda.gov/catalog/645415> doi:

https://doi.org/10.1111/j.1748-5827.2003.tb00101.x

CASTRO, M. C. M. D.; PEREYRA, W. J. F. FILHO, L. N. O.; GUAXUPÉ, C. A. R.; SOUSA, N. A. D.; SOUSA, A. A. D.Tumor de glândula ceruminosa com invasão intracraniana: relato de caso. Arquivos de Neuro-Psiquiatria, v. 58, n.2, p.324329. 2000.

Disponível em:

https://www.scielo.br/j/anp/a/fRwdYLbtKPS9rS7YpMDhDrr/> doi: https://doi.org/10.1590/S0004-282X2000000200020

COSTA, A. L.; CAPELLA, S. D. O.; VIVES, P.; FERNANDES, C. G.; NOBRE, M. D. O. Carcinoma de glândulas ceruminosas na otite canina. Medvep - Revista Científica de Medicina Veterinária, Pequenos Animais e Animais de Estimação; v.2, n.48, p.19-24., 2018. Disponível em: < https://medvep.com.br/carcinoma-deglandulas-ceruminosas-na-otite-canina/>

DIAZ, V. M. M. Adenocarcinoma de glándulas ceruminosas en un canino: reporte de caso. Revista Medicina Veterinária, Bogotá, v.37, n.11, p.95-102., July/Dec. 2018. Disponível em: https://www.researchgate.net/publication/331678267 Adenocarcinoma de glandula s_ceruminosas_en_un_canino_reporte_de_caso> doi: 10.19052/mv.vol1.iss37.11

GRANDI, F.; RONDELLI, M. C. H. Neoplasias Cutâneas. In: DALECK, C. R.; DE NARDI, A. B. Oncologia em Cães e Gatos. $2^{\underline{a}}$ ed. Rio de Janeiro: Editora Roca, $p$. 339-363, 2017.

KUBBA, M. A.G.; WAFA, S. N.; AZREG, S. A. A. L. Ceruminous gland adenocarcinoma in a domestic Persian-mix cat (Felis catus). Open Veterinary Journal, v.8, n.2, p.168-171, 2018. Disponível em: <https://pubmed.ncbi.nlm.nih.gov/29805962/ > doi: 10.4314/ovj.v8i2.9

MEDEIROS, V. B.; REIS, P. F. C. D. C.; FILGUEIRA, K. D. Carcinoma de glândulas ceruminosas em um felino doméstico. Medvep dermatologia, São Paulo, v. 3, n.8, p. 48-49, 2014. Disponível em: < https://www.bvsvet.org.br/vetindex/periodicos/medvep-dermato/3-(2014)-8/carcinoma-de-glandulasceruminosas-em-um-felino-domestico/>

NELSON, R. W.; COUTO, C. G. Medicina interna de pequenos animais. 5. ed. Rio de Janeiro, 2021.1440p.

PAVLETIC. M. M.; Partial vertical ear canal resection in two cats. Journal of the American Veterinary Medical Association. Boston, v. 255, n. 12, p. 1365-1368, Dec/2019. Disponível em:< https://pubmed.ncbi.nlm.nih.gov/31793833/> doi: 10.2460/javma.255.12.1365

TAYLOR, S. M. Inclinação da cabeça. In: NELSON, R. W.; COUTO, G. C. Medicina Interna de Pequenos animais. 3 ed., Rio de Janeiro: Elsevie, cap. 70, p. 969-973, 2006. 